\title{
Intravital autofluorescence 2-photon microscopy of murine intestinal mucosa with ultra-broadband femtosecond laser pulse excitation: image quality, photodamage, and inflammation
}

Antje Klinger

Lisa Krapf

Regina Orzekowsky-Schroeder

Norbert Koop

Alfred Vogel

Gereon Hüttmann

\section{SPIE.}




\title{
Intravital autofluorescence 2-photon microscopy of murine intestinal mucosa with ultra-broadband femtosecond laser pulse excitation: image quality, photodamage, and inflammation
}

\author{
Antje Klinger, ${ }^{\mathrm{a}, \dagger}$ Lisa Krapf, ${ }^{\mathrm{b}, \dagger}$ Regina Orzekowsky-Schroeder, ${ }^{\mathrm{b}}$ Norbert Koop, ${ }^{\mathrm{b}}$ Alfred Vogel, ${ }^{\mathrm{b}}$ and \\ Gereon Hüttmann ${ }^{b, c, *}$ \\ aUniversity of Lübeck, Institute of Anatomy, Ratzeburger Allee 160, 23562, Lübeck, Germany \\ bUniversity of Lübeck, Institute of Biomedical Optics, Peter-Monnik-Weg 4, 23562, Lübeck, Germany \\ ${ }^{c}$ Airway Research Center North (ARCN), Member of the German Center for Lung Research (DZL), Germany
}

\begin{abstract}
Ultra-broadband excitation with ultrashort pulses may enable simultaneous excitation of multiple endogenous fluorophores in vital tissue. Imaging living gut mucosa by autofluorescence 2-photon microscopy with more than $150 \mathrm{~nm}$ broad excitation at an 800-nm central wavelength from a sub-10 fs titanium-sapphire (Ti:sapphire) laser with a dielectric mirror based prechirp was compared to the excitation with $220 \mathrm{fs}$ pulses of a tunable Ti:sapphire laser at 730 and $800 \mathrm{~nm}$ wavelengths. Excitation efficiency, image quality, and photochemical damage were evaluated. At similar excitation fluxes, the same image brightness was achieved with both lasers. As expected, with ultra-broadband pulses, fluorescence from $\mathrm{NAD}(\mathrm{P}) \mathrm{H}$, flavines, and lipoproteins was observed simultaneously. However, nonlinear photodamage apparent as hyperfluorescence with functional and structural alterations of the tissue occurred earlier when the laser power was adjusted to the same image brightness. After only a few minutes, the immigration of polymorphonuclear leucocytes into the epithelium and degranulation of these cells, a sign of inflammation, was observed. Photodamage is promoted by the higher peak irradiances and/or by nonoptimal excitation of autofluorescence at the longer wavelength. We conclude that excitation with a tunable narrow bandwidth laser is preferable to ultra-broadband excitation for autofluorescence-based 2-photon microscopy, unless the spectral phase can be controlled to optimize excitation conditions. () The Authors. Published by SPIE under a Creative Commons Attribution 3.0 Unported License. Distribution or reproduction of this work in whole or in part requires full attribution of the original publication, including its DOI. [DOI: 10.1117/1.JBO.20.11.116001]
\end{abstract}

Keywords: 2-photon microscopy; autofluorescence; photodamage; inflammation.

Paper 150357RR received Jun. 3, 2015; accepted for publication Sep. 16, 2015; published online Nov. 2, 2015.

\section{Introduction}

Nonlinear microscopy has opened a new door to intravital imaging. Selective excitation of fluorescence by 2-photon absorption visualizes biological processes in animals and humans in real time as they occur in their native environment. ${ }^{1}$ Especially immunological questions benefit from this technology, as most immune cells are highly motile and interact extensively with each other and their environment. ${ }^{2}$ Autofluorescence 2-photon (A2P) microscopy offers the ability for high-resolution subcellular imaging based only on intrinsic molecular signals in cells, such as NAD $(\mathrm{P}) \mathrm{H}$ [reduced nicotinamide adenine dinucleotide(phosphate) and flavin adenine dinucleotide (FAD)], and reveals most, if not all, of the cells and tissues of the small intestinal mucosa. ${ }^{3}$ As there are no additional fluorescent markers applied to the tissue, A2P microscopy may in the future be used for clinical diagnosis of tumors or other skin diseases. ${ }^{4,5}$ For A2P imaging, commonly tunable titanium-sapphire (Ti:sapphire) lasers with pulse lengths around $100 \mathrm{fs}$ are utilized. The laser wavelength has to be changed between 720 and $850 \mathrm{~nm}$ for optimal excitation of naturally occurring fluorophores, such as

*Address all correspondence to: Gereon Hüttmann, E-mail: huettmann@ bmo .uni-luebeck.de

†These authors contributed equally.
$\mathrm{NAD}(\mathrm{P}) \mathrm{H}$, flavines, lipopigments, and second-harmonic generation (SHG) of collagen. ${ }^{6}$ If additional fluorescent proteins or exogenous dyes are employed, an even larger tuning range may be useful. ${ }^{7,8}$ For optimal imaging of multiple fluorophores, the specimen has to be scanned several times with different excitation wavelengths at the expense of reduced imaging speed and increased motion artifacts. Alternatively, a single wavelength can be chosen to excite all fluorophores simultaneously but at a significantly reduced efficiency for most fluorophores. Compact and rugged Ti:sapphire lasers with pulse widths below 20 fs are available which have a bandwidth spanning over more than $100 \mathrm{~nm}$. With properly corrected group delay dispersion, an increase of excited fluorescence due to the higher peak irradiance of such laser pulses was demonstrated. ${ }^{9}$ The dyes Indo-1, FITC, and TRITC showed a 1.6 to 6.7 increase in fluorescence when simultaneously excited with ultrashort pulses with a $125-\mathrm{nm}$ spectral width. ${ }^{9}$ Fluorescence in cells and tissues was successfully imaged with a 30 -fs pulse width after compensation of dispersion by a prism pair. ${ }^{10}$ Moreover, tissue autofluorescence [NAD $(\mathrm{P}) \mathrm{H}$ and FAD] and SHG from collagen could be imaged simultaneously with A2P. With a $100 \mathrm{~nm}$ bandwidth around a center wavelength of $780 \mathrm{~nm}$ and a pulse width below 13 fs, the autofluorescence of skin has been imaged ex vivo. ${ }^{11}$ However, in that study, the excitation flux was not decreased. Instead, twice the radiant flux compared 
to excitation at $780 \mathrm{~nm}$ with $150 \mathrm{fs}$ pulses had to be used for similar image quality. Here, the overlap between the broad excitation spectrum and the absorption of $\mathrm{NAD}(\mathrm{P}) \mathrm{H}$ and other fluorophores was poor and obviously not compensated by the increased peak irradiance of the shorter pulse width. The lower excitation efficiency could be compensated by increasing the excitation power, but no information was given on how this would affect the rate of photodamage, which is a severe limit in long-time in vivo microscopy.

Different mechanisms contribute to the photodamage of cells and tissue. ${ }^{12}$ One-photon absorption by endogenous chromophores including water may cause damage by photochemical changes or heating of the tissue. ${ }^{12}$ This type of damage increases linearly with the radiant flux and can be reduced in relation to 2-photon excited fluorescence using shorter pulses. The 2-photon excitation of fluorophores, which causes the observed fluorescence, may lead to a change of redox states, the production of singlet oxygen, or other reactive oxygen species (ROS). ${ }^{12}$ Thus the damage caused scales exactly in the same way as the fluorescence. Pulse width and pulse frequency will not have an influence on the ratio between the fluorescence and this type of tissue damage. 2-photon excitation of molecules that do not contribute to the detected autofluorescence may as well add to photodamage. This contribution also scales with the radiant flux squared, but its wavelength dependence differs from that of the fluorescence. It can be minimized by optimizing the overlap between 2-photon absorption of the fluorophore and the excitation wavelengths. Finally, excited state absorption and multiphoton excitation caused by more than two photons may lead to unwanted side effects that increase more rapidly with the irradiance than the fluorescence. ${ }^{12}$ This may include direct absorption of DNA by a 3 -photon process ${ }^{13}$ and the photochemistry caused by the generation of free electrons through the multiphoton excitation of water. ${ }^{14}$ Both processes are promoted by a shorter pulse width and shorter wavelength of the exciting pulses.

The dominant mechanism for photodamage will determine the optimal choice of pulse parameters. If linear mechanisms need to be avoided, short pulses and low repetition rates with higher peak irradiances are preferred. In contrast, if the dominant damage mechanism exhibits a power dependence with an exponent larger than two, as observed, for example, by Hopt and Neher, ${ }^{15}$ longer pulses and high repetition rates are favored for damage-free fluorescence imaging. There is some evidence that at pulse widths above $100 \mathrm{fs}$, relevant photodamage is mainly produced by a 2-photon process. ${ }^{16,17}$ Reducing the pulse width in general will promote higher-order absorption and might, therefore, lead to additional damage.

In A2P microscopy, it is not the available laser power that restricts the brightness and imaging speed, but rather photochemical destruction of tissue and the resulting biological effects. ${ }^{7}$ Therefore, a comparison of different excitation schemes to find optimal conditions for intravital imaging has to consider tissue damage. Especially in time-lapse imaging of mucosal tissue, the avoidance of tissue damage is of utmost importance when migratory behavior and cell-cell and cell-matrix interactions are to be observed under physiological conditions. Several endogenous and exogenous stimuli have been shown to cause the release of pro-inflammatory cytokines and the recruitment of immune cells. ${ }^{18}$ Therefore, optimal excitation wavelengths, the highest possible detection efficiency, and the minimal possible scanning time should be used.
The total amount of fluorescence (i.e., number of detected photons) until the biological system is damaged or altered in its biological function, is a key parameter for comparing the suitability of different lasers or irradiation parameters. Optimizing this parameter will increase the image quality and/or the observation time of $\mathrm{A} 2 \mathrm{P}$ intravital imaging.

In this study, we compare two excitation regimes. The use of ultra-broadband pulses $(>150 \mathrm{~nm})$ produced by a sub-10 fs Ti: sapphire laser with compensation of the linear chirp to enhance peak power, with excitation by 220 fs pulses from a commonly utilized tunable Ti:sapphire laser (10 nm spectral width). Both lasers were used in the same experimental setting for A2P microscopy of small intestinal mucosa. Whereas for ultra-broadband excitation, pulse durations were shortened by precompression with commercially available chirped mirrors to a value below $100 \mathrm{fs}$; for the latter system, the pulse width in the sample was approximately 220 fs since no prechirp was applied. The relationship between the fluorescence and photodamage was investigated by acquiring image sequences of apical and basal cytoplasm of the villus epithelium with different excitation fluxes. The effects of two excitation wavelengths (730 and $800 \mathrm{~nm}$ ) from the tunable system were compared to ultra-broadband excitation. At $730 \mathrm{~nm}, \mathrm{NAD}(\mathrm{P}) \mathrm{H}$ was excited near the maximum of its 2-photon absorption spectrum. Alternatively, a wavelength of $800 \mathrm{~nm}$ was chosen for FAD imaging and for evaluating the effect of exciting $\mathrm{NAD}(\mathrm{P}) \mathrm{H}$ at its long-wavelength absorption edge. The total number of detected fluorescence photons per pixel before the onset of photodamage was used to compare the photodamage potential of both lasers quantitatively. Two biological phenomena were used as criteria for photodamages: first, the sudden increase of fluorescence intensity, often called hyperfluorescence ${ }^{6,7,19}$ and second, the invasion and degranulation of polymorphonuclear leucocytes into the irradiated area. Using laser pulses shorter than $10 \mathrm{fs}$ as input to the microscope, the group delay dispersion of the optical components will significantly influence the pulse width in the sample. The expected linear increase of the fluorescence, as the pulse duration is reduced, will only be observed when higher dispersion orders are carefully corrected. ${ }^{20}$ A full compression to transform-limited pulses needs special equipment for controlling the spectral phase and a full characterization of ultrashort laser pulses, ${ }^{21}$ which are not a standard in intravital imaging laboratories due to cost and complexity. Instead of rigorously investigating the influence of pulse width on fluorescence intensity and photodamage, the aim of our experimental work was to evaluate the usefulness of a commercially available broadband ultrashort Ti:sapphire laser with a dielectric mirror based prechirp for autofluorescence-based 2-photon microscopy.

\section{Materials and Methods}

\subsection{Mouse Model}

Female Balb/c mice $(n=5)$, and C57BL/6 mice $(n=5) 8$ to 10 weeks of age, were purchased from Charles River Laboratories (Sulzfeld, Germany) and kept under standard conditions with free access to food and water. Animals were anesthetized with a combination of Fentanyl (Bayer, Leverkusen Germany), Midazolam (Curamed, Karlsruhe, Germany), and Medetomidin (Pfizer, Karlsruhe, Germany) injected intraperitoneally. After assuring adequate anesthesia, the abdominal cavity was opened and an isolated ileal loop was gently protruded without 
disturbing the blood supply. The loop was glued (3M Vetbond TissueAdhesive, St. Paul, Minnesota) on a heated metallic block and sliced carefully so that the mucosa could be gently fixed to a microscopic cover slip to dampen motion artifacts, while still maintaining tissue viability. ${ }^{3,6}$ During all procedures, the small intestine was constantly moisturized with saline, and the core body temperature was maintained at $37^{\circ} \mathrm{C}$ using a homeothermic table. All animal experiments were approved by the state government (Ministerium für Umwelt, Naturschutz und Landwirtschaft Schleswig-Holstein V742-72241.122, TVNo. 23/p/04, V312-72241.122-1 (4-1/10), TV-No. 23/1f/10).

\subsection{Autofluorescence 2-Photon Microscopy}

Imaging with both lasers was compared at the same instrument, the multiphoton tomograph Derma Inspect 101 (JenLab, Jena, Germany). A tunable femtosecond Ti:sapphire laser (Mai Tai Spectra Physics, Mountain View, California) with a pulse width below 100 fs and $80 \mathrm{MHz}$ repetition frequency was used for imaging at the excitation wavelengths 730 and $800 \mathrm{~nm}$. As is usually done, the group delay dispersion (GDD) of the microscope and objective was not compensated, which increased the pulse width from below 100 fs to approximately $220 \mathrm{fs}$. This value was calculated from the measured pulse width of the Mai Tai and the GDD which was determined separately for the microscope objective and the scanning optics. A transform limited output of the Mai Tai was assumed. Since both, laser pulse width and GDD dropped between $730 \mathrm{~nm}$ and $800 \mathrm{~nm}$, the pulse width changed less than $10 \%$ in this range.

In addition, sub-10 fs broadband Ti:sapphire laser (Integral Pro, Femtolasers Produktions $\mathrm{GmbH}$ ) with a $85-\mathrm{MHz}$ repetition was additionally coupled to the Derma Inspect using a flipping mirror [Fig. 1(a)]. A dielectric mirror based prechirp unit (Mosaic Pro V, Femtolasers Produktions $\mathrm{GmbH}$ ) compensated second-order dispersion of the optical components. The overall GDD in the light path was determined to be $7950 \mathrm{fs}^{2}$ for the $800 \mathrm{~nm}$ wavelength (Table 1). This corresponded to the maximum negative GDD of the prechirp unit and could thus be compensated. Pulse duration was measured in the focal volume by an autocorrelator (Femtometer, Femtolasers Produktions
Table 1 Calculated efficiency of 2-photon excitation of $\mathrm{NAD}(\mathrm{P}) \mathrm{H}$ and flavin adenine dinucleotide (FAD) with tunable and broadband pulses.

\begin{tabular}{lccc} 
& $\begin{array}{c}\text { Mai Tai, } \\
730 \mathrm{~nm}\end{array}$ & $\begin{array}{c}\text { Mai Tai, } \\
800 \mathrm{~nm}\end{array}$ & $\begin{array}{c}\text { Integral (794 nm } \\
\text { center wavelength) }\end{array}$ \\
\hline $\begin{array}{l}\text { Pulse duration } \\
\text { laser output (fs) }\end{array}$ & 105 & 90 & $<6$ \\
$\begin{array}{l}\text { Group dispersion } \\
\text { delay (fs }{ }^{2} \text { ) }\end{array}$ & 7200 & 6400 & 7500 \\
$\begin{array}{l}\text { Pulse duration (fs) } \\
\text { 2-photon excitation } \\
\text { cross-section } \sigma_{2 P} \\
\text { for NAD(P)H (GM) }\end{array}$ & 0.041 & 0.0012 & 0.014 \\
$\begin{array}{l}\text { Normalized excitation } \\
\sigma_{2 P} / \lambda f \tau\end{array}$ & 1 & 220 (calc.) & 10 (optimal \\
GDD correction)
\end{tabular}

$\mathrm{GmbH})$. The autocorrelation traces showed that the pulses are not transform limited. Though the autocorrelator software determined a pulse width of around $10 \mathrm{fs}$ from the central peak, the increased asymptotic level and subpulses at 80 fs indicate considerably longer pulses, probably above 15 fs [Fig. 2(b)]. A pulse duration below $10 \mathrm{fs}$, which is supported by a spectral width of $150 \mathrm{~nm}$, is only reached when the higher-order GDD is compensated, which was beyond our technical means.

The small intestine was imaged by A2P microscopy using a $40 \times / 1.2$ water immersion objective (Carl Zeiss Microscopy $\mathrm{GmbH}$, Jena, Germany). Emission between 380 and $680 \mathrm{~nm}$ (a)

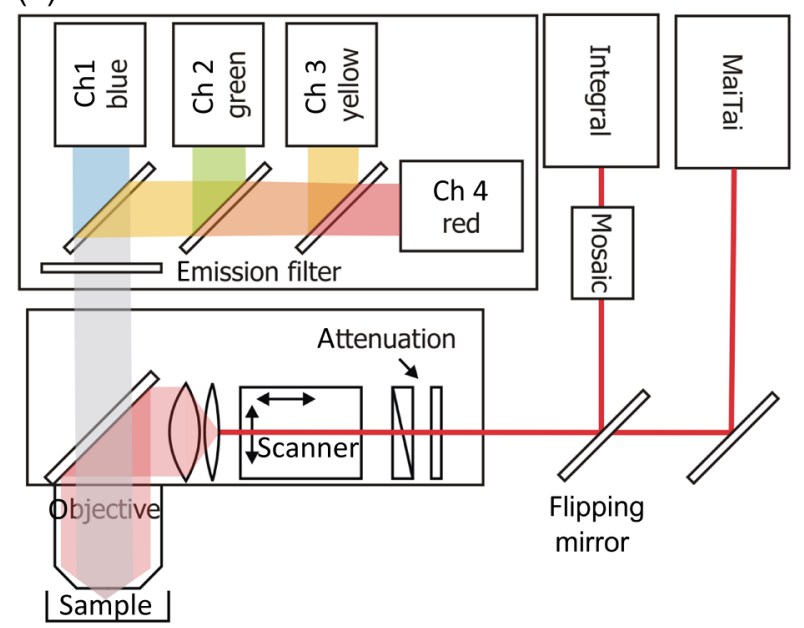

(b)

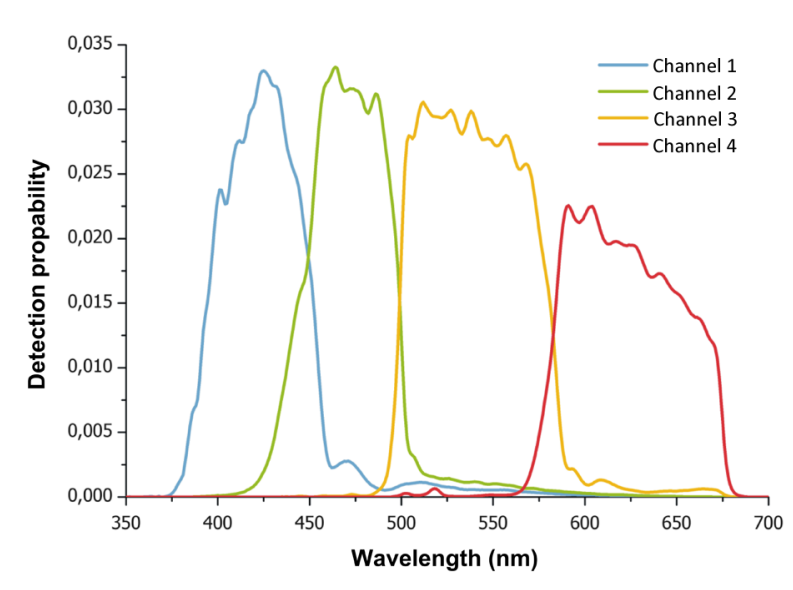

Fig. 1 (a) Schematic drawing of the 2-photon microscope with fluorescence detection in four spectral channels and (b) detection probability of the spectral channels including the collection yield of the objective, the transmission of all optical elements, and the quantum efficiency of the detectors. 
(a)

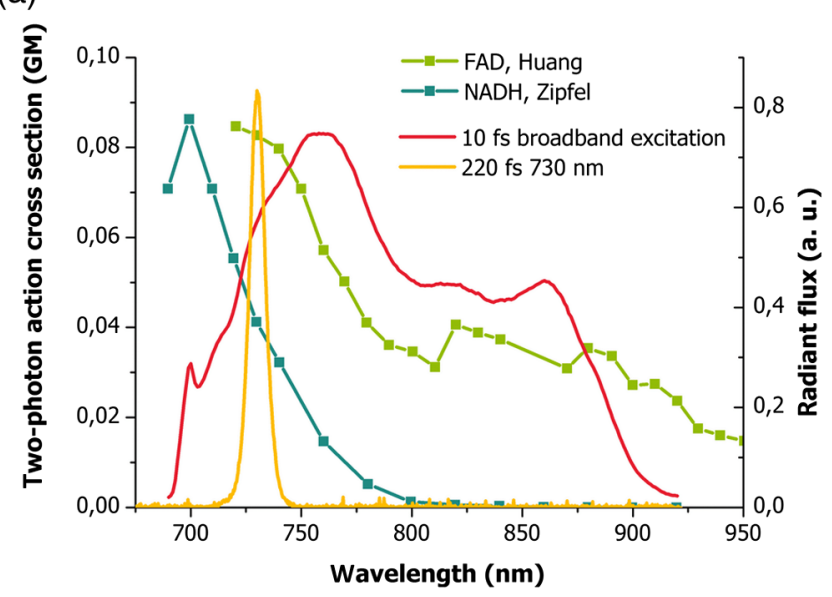

(b)

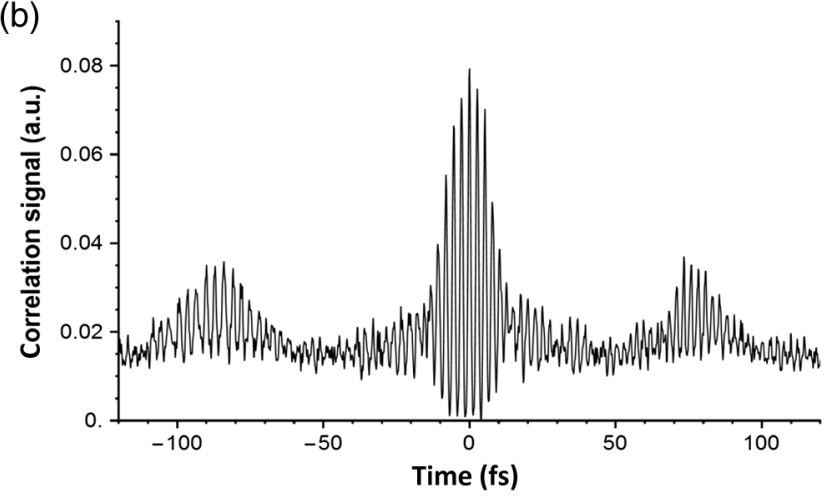

Fig. 2 (a) Spectral dependence of the 2-photon action cross sections of $\mathrm{NAD}(\mathrm{P}) \mathrm{H}^{7}$ and FAD. ${ }^{22}$ For comparison, the spectrum of the Spectra Physics Mai Tai (220 fs pulse) and the Femtolasers Integral Pro (10 fs pulse) are also shown. (b) Autocorrelation trace of the pulses from Femtolasers Integral Pro measured with a second-harmonic generation (SHG) signal inside the sample volume.

was detected in four spectral channels. Detection efficiencies are shown in Fig. 1(b). Channel 4 was not included in data evaluation because a significant amount of scattered excitation light was picked up by the detector and the fluorescence signal in that channel was very weak.

The relation between gray values registered by the PMTs and the corresponding photon numbers was established by calibration measurement. For this purpose, images of homogeneously fluorescing plastic slides (Chroma Chroma Technology $\mathrm{GmbH}$, Olching, Germany) were acquired with different excitation intensities, and the mean intensity and variance of fluorescence emission values were evaluated. For Poisson distributed noise, there is a linear correlation between variance and mean fluorescence intensity values. Their ratio was used as the conversion factor between the gray value and number of detected photons. ${ }^{23}$ Using these factors, fluorescence intensities of the autofluorescence images were converted into photon numbers per pixel.

\subsection{Comparison of the Photodamage Potential of Both Laser Systems}

The time-averaged rate of photon detection $\langle F(t)\rangle$ for 2-photon excitation depends on the 2-photon action cross-section $\sigma_{2 P}$, concentration $c$ of the excited fluorophores, time-averaged radiant flux $\langle P(r, t)\rangle$, wavelength $\lambda$, pulse duration $\tau$, and repetition rate $f$ of the excitation: ${ }^{24}$

$\langle F(t)\rangle \propto \sigma_{2 P} c \frac{\langle P(r, t)\rangle^{2}}{\lambda f \tau}$.

We introduce the fluorescence detection rate normalized by the excitation flux squared

$\frac{\langle F(t)\rangle}{\langle P(r, t)\rangle^{2}} \propto \frac{\sigma_{2 P}}{\lambda f \tau}$

as an excitation-flux-independent measure of the 2-photon excitation efficiency for the fluorescent species under consideration.

For a quantitative comparison of the damage potential of both lasers, image sequences were taken at different excitation fluxes in the apical and basal part of the villus epithelium of the mouse intestine. Imaged areas covered a field of $120 \times 120 \mu \mathrm{m}^{2}$ at a pixel size of $0.23 \times 0.23 \mu \mathrm{m}^{2}$.

Time-lapse series of $100 \mathrm{~A} 2 \mathrm{P}$ images were recorded at intervals of $7.4 \mathrm{~s}$. Each series covered a time of $14 \mathrm{~min}$. In order to evaluate the average brightness of each fluorescence image in the three spectral channels, the mean number of photons from 10 regions of interest (ROI) in the cytoplasm was determined (Fig. 3).

The time for the onset of hyperfluorescence was measured during irradiation of the apical cytoplasm. When hyperfluorescence started, image acquisition was stopped after a few more images, as the hyperfluorescent lesions grew fast, destroyed large tissue areas, and led to saturation of the detector signal (Fig. 5). The total number of fluorescence photons before the onset of hyperfluorescence was calculated by multiplying the mean photon number per pixel with the number of images that could be acquired before hyperfluorescence started (Fig. 6).

The immigration of inflammatory cells into the irradiated tissue was evaluated during and after irradiation of the villus epithelium as a sensitive physiological marker of tissue damage. In the irradiation series of the basal part of the epithelium, polymorphonuclear leucocytes (PMNLs) could occasionally be observed migrating into the irradiated area. When the apical part of the villus epithelium was imaged, no inflammatory cells were observed, as inflammatory cells can probably move only in the basal part of the epithelium due to tight junctions and desmosomes in the apical part of the cells. In order to quantify the immune response, image stacks of the irradiated villi were acquired up to one hour after the irradiation to screen the tissue for immunological reactions. The fraction of irradiation sequences taken at the basal cytoplasm and the fraction of image stacks of the villi, in which the immigration of immune cells was observed, was counted for assessing the tissue damage (Fig. 9).

\subsection{Image Analysis}

The image brightness in the cellular compartments and ROIs was evaluated with the software ImageJ. ${ }^{25}$ Pixel values were corrected for background signal by subtraction of a dark image. The resulting intensities were converted into detected photon numbers as described in Sec. 2.2.

Stacks and time series were processed using the software Imaris version 7.2.0 (Bitplane, Zurich, Switzerland). For cell tracking, image series were transformed into volume-rendered movies, and spot analysis was used for manual tracking of 

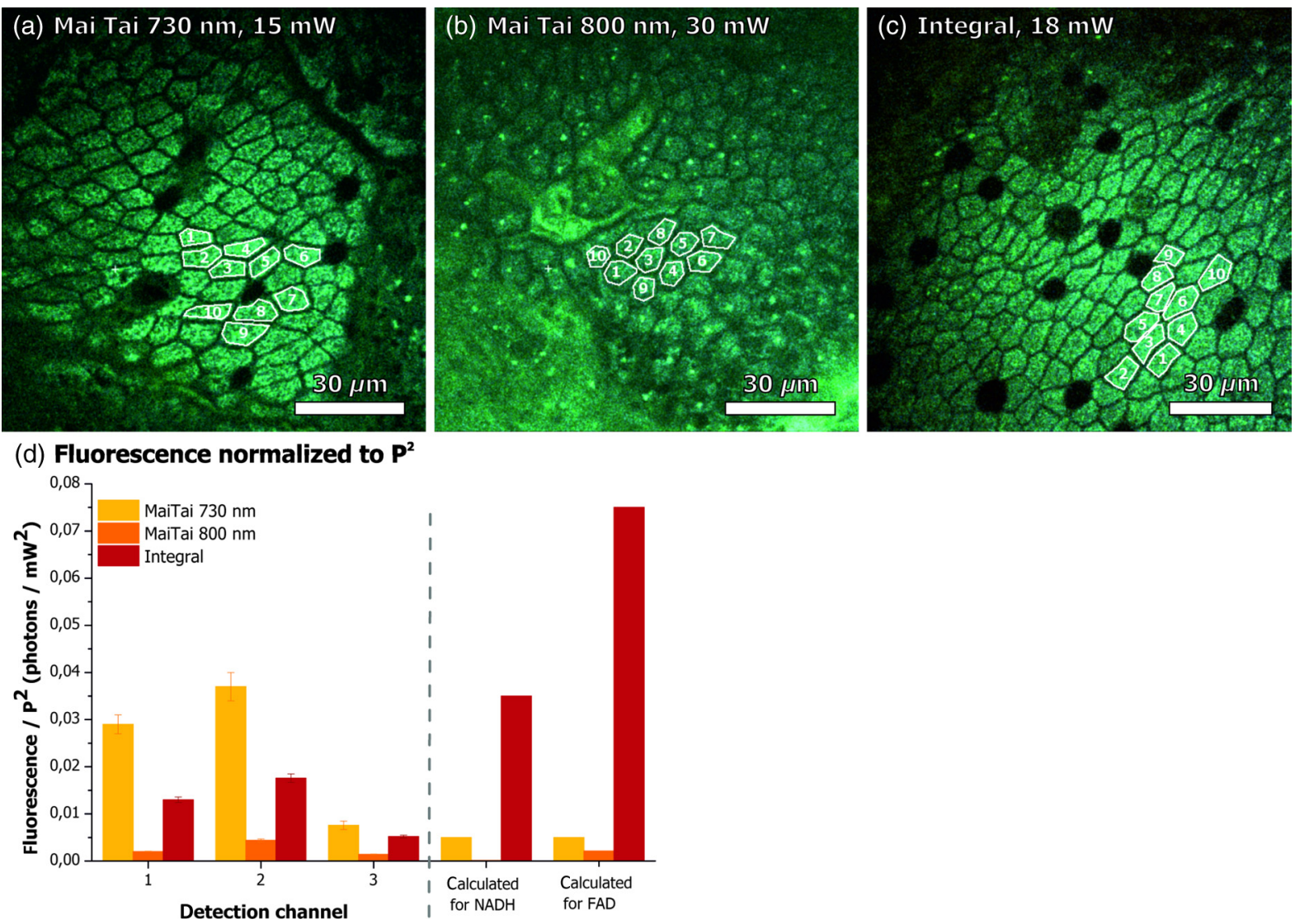

Fig. 3 Autofluorescence images of a plane through the apical cytoplasm of villus epithelium. Images in (a) and (b) were acquired using 220 fs pulses with excitation wavelengths of 730 and $800 \mathrm{~nm}$, respectively. The image in (c) was recorded with ultra-broadband pulses. In each image, regions of interest (ROIs) were used for the determination of image brightness. (d) Fluorescence in the three spectral channels divided by the squared excitation flux. For comparison, calculated normalized fluorescence from Table 1 is shown in arbitrary units.

cell motility. The calculations of the cell velocity, straightness, track length, and displacement were performed using the algorithms provided by Imaris.

\section{Results and Discussion}

\subsection{Efficiency of Autofluorescence Excitation}

The 2-photon action cross-section $\sigma_{2 P}$ of $\mathrm{NAD}(\mathrm{P}) \mathrm{H}$ provides the main contribution to the cellular autofluorescence of epithelial enterocytes in the small intestine. ${ }^{7}$ Between 730 and $800 \mathrm{~nm}$, the 2-photon excitation of $\mathrm{NAD}(\mathrm{P}) \mathrm{H}$ drops by more than an order of magnitude (Fig. 2). In contrast, for the same spectral shift of excitation, the second relevant chromophore FAD looses only a little more than $50 \%$ of its absorption. Table 1 compares the expected efficiency of the 2-photon excitation of $\mathrm{NAD}(\mathrm{P}) \mathrm{H}$ and FAD with tunable 220 fs pulses at 730 and $800 \mathrm{~nm}$ to the excitation efficiency with ultra-broadband pulses if they were compressed to $10 \mathrm{fs}$. The 2-photon action-crosssection $\sigma_{2 P}$ for these pulses was calculated based on the fluorophore spectra and the laser emission spectrum shown in Fig. 2. Excitation-normalized values of the expected fluorescence were obtained by relating $\sigma_{2 P}$ to the pulse duration, repetition rate, and wavelength (center wavelength for the ultra-broadband pulses). In order to facilitate the comparison between the different excitation modalities, all $\left(\sigma_{2 P} / \lambda f \tau\right)$ values were, in turn, normalized by the value for $730 \mathrm{~nm} / 220 \mathrm{fs}$ excitation.
According to the data in Table 1, excitation of NAD(P)H should be eight times more efficient with $10 \mathrm{fs}$ ultra-broadband pulses than with $220 \mathrm{fs}$ pulses at a wavelength of $730 \mathrm{~nm}$. The higher irradiance of 10 fs pulses should more than compensate the lower spectral overlap between the 2-photon absorption and excitation. Excitation of $\mathrm{NAD}(\mathrm{P}) \mathrm{H}$ at $800 \mathrm{~nm}$ should be 40 times less efficient compared to $730 \mathrm{~nm}$ and even 300 times less efficient than with broadband pulses compressed to $10 \mathrm{fs}$. Excitation of FAD is expected to be only 14 times more efficient than with $730 \mathrm{~nm} / 220 \mathrm{fs}$ pulses.

These predictions were experimentally tested by in vivo imaging of the apical villus epithelium (Fig. 3). As expected, fluorescence was drastically reduced when the excitation wavelength of $220 \mathrm{fs}$ pulses was tuned from 730 to $800 \mathrm{~nm}$. Twice the excitation flux had to be used to get an acceptable image quality. Enterocytes were visible at both wavelengths, while lysosomes appeared more pronounced at the $800 \mathrm{~nm}$ excitation. Since a pulse compression to 10 fs was not achieved, the use of ultra-broadband pulses did not lower the required excitation flux compared to $730 \mathrm{~nm} / 220 \mathrm{fs}$ excitation. Thus, at comparable image quality, a similar light exposure (see Fig. 3) was needed.

For quantitative evaluation of the fluorescence intensities, 10 ROIs inside the apical cytoplasm of an enterocyte were evaluated for each excitation condition. The mean photon number per pixel was calculated [Fig. 3(d)]. Instead of a 7 to 15 -fold 
increase of excitation-normalized fluorescence, which is expected for a $10 \mathrm{fs}$ pulse width, the ultra-broadband excitation with our not fully compressed pulses generated for the same excitation power slightly less fluorescence than 220 fs pulses at $730 \mathrm{~nm}$. Compared to $800 \mathrm{~nm}$ excitation, the normalized fluorescence excited by ultra-broadband pulses increased only 4-fold instead of the 300 times calculated for $10 \mathrm{fs}$ pulse width.

This discrepancy corroborates the incomplete GDD compensation seen in the autocorrelation traces [Fig. 2(b)]. Generation of pulse widths below $10 \mathrm{fs}$ (with a spectral width of over $150 \mathrm{~nm}$ ) in the microscope focus requires an exact compensation of the GDD introduced by the highly dispersive microscope objectives. This is only achieved if higher-order dispersion compensation is employed. Advanced control of the spectral phase of broad pulse using, for example, a multiphoton intrapulse interference phase scan (MIIPS) would be needed to reach the 2-photon excited fluorescence and SHG levels that are predicted by Eqs. (1) and (2) at these bandwidths. At a spectral width of $30 \mathrm{~nm}$, a deviation from the linear increase of the detected emission with spectral width or the inverse pulse duration was already observed. ${ }^{20}$ Since a five times broader spectral width was used in these experiments with first-order GDD compensation, fluorescence levels are significantly lower than expected for bandwidth-limited pulses. Increased pulse width and intensity in the side lobes did not optimally contribute to fluorescence excitation. Since the excitation efficiency depends in a complex manner on the pulse chirp, ${ }^{26}$ an exact calculation of the excitation efficiency would have to incorporate the spectral phase, which was not measured. The short-wavelength spectral components, which have the highest deviation from a constant GDD and also the highest absorption by the fluorophores, are not optimally corrected and will, therefore, contribute much less to excitation than assumed in Table 1. Additionally longitudinal chromatic aberration, which was approximately $1 \mu \mathrm{m}$ over the wavelength range from 700 to $900 \mathrm{~nm}$ for the objective used, also prevents a perfect overlap of all wavelength components in the sample and may further reduce the excitation efficiency.

In conclusion, the differences between the calculated and measured excitations of cellular autofluorescence reflect deficiencies in the setup. Increased technical efforts are needed for optimal compensations of GDD and chromatic aberrations. If successfully done, a reduction of the excitation flux by one order of magnitude can be expected. However, as will be shown below, the speed and quality of imaging are not only limited by the excitation power, but also by photodamage. Hence, no efforts were made to increase the excitation efficiency in our setup. In contrast, in a clinical setting, where A2P microscopy with fixed optics will be used, specifically tailored dispersive mirrors could be used to exactly compensate higher-order GDD and attain optimum excitation efficiency. ${ }^{27}$

A series of images were taken at different depths within the small intestinal mucosa from the apical cytoplasm of the epithelium down to the lamina propria to compare $220 \mathrm{fs} / 730 \mathrm{~nm}$ excitation with ultra-broadband excitation (Fig. 4, Video 1 and Video 2). Mitochondria containing $\mathrm{NAD}(\mathrm{P}) \mathrm{H}$ as the main fluorophore are equally visible in both cases. FAD in lysosomes and antigen presenting cells, which has a red-shifted absorption, ${ }^{3}$ was considerably more visible with ultra-broadband excitation due to the good overlap with the emission spectrum. Hence, the ultra-broadband pulses revealed the combined information of 730 and $800 \mathrm{~nm}$ excitation in just one scan. SHG by collagen is efficient above $800 \mathrm{~nm}$ and should also be visible with ultra-broadband excitation. However, it was not observed in the present experiments due to a lack of SHG active type I collagen in the small intestine.
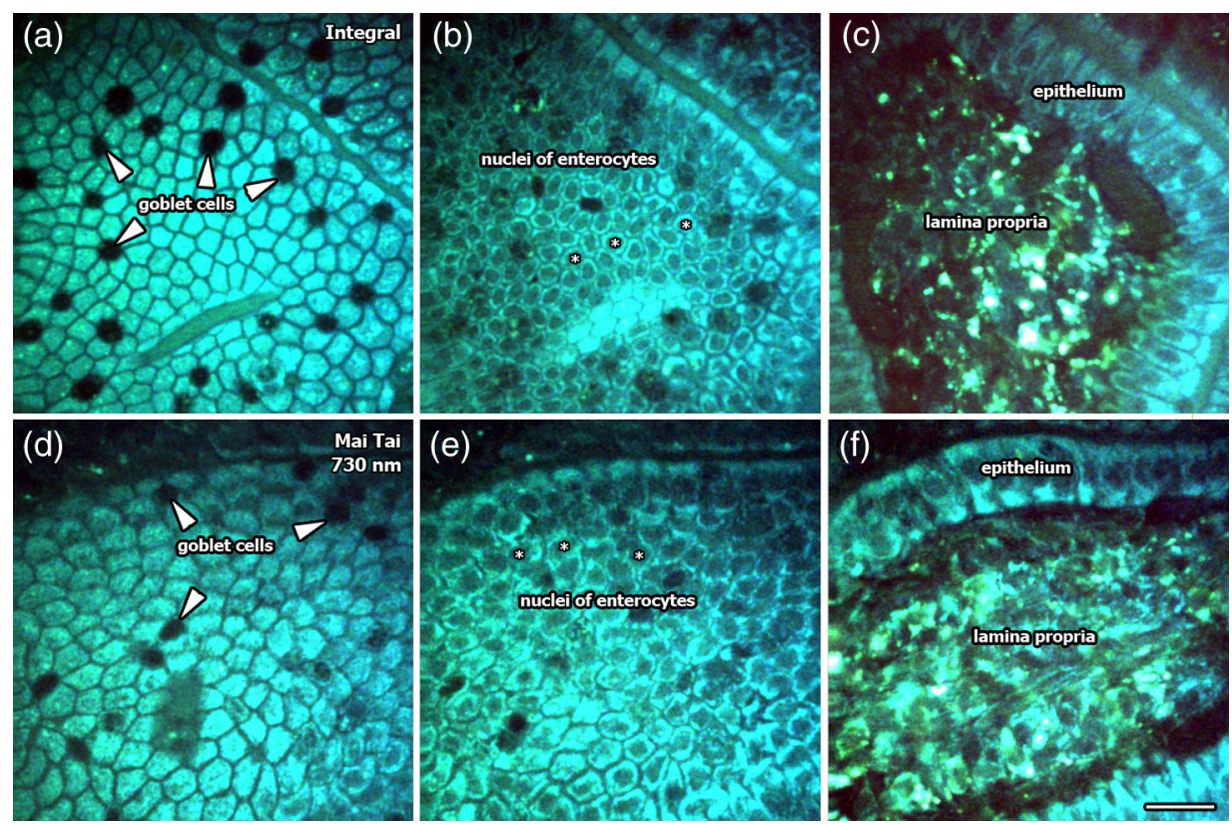

Fig. 4 (a, d) Autofluorescence 2-photon (A2P) images of the apical cytoplasm. Autofluorescence was mainly from $\mathrm{NAD}(\mathrm{P}) \mathrm{H}$ in mitochondria of enterocytes. Mucus of goblet cells (arrows) appears dark. (b, c) The basal part of the epithelial cells. The cells nuclei (asterisks) are surrounded by a small rim of bright cytoplasm. (c, f) Epithelium and the underlying lamina propria with many different cells. The increased spectral bandwidth of the sub-10 fs Integral Pro laser shows lysosomes, macrophages, and other FAD-containing structures with good contrast (scale bar, $20 \mu \mathrm{m}$ ) (Video 1, MPEG, $3.91 \mathrm{MB}$; Video 2, MPEG, 4.15 MB) [URL: http://dx.doi.org/10.1117/1.JBO.20.11.116001.1]. [URL: http://dx.doi. org/10.1117/1.JBO.20.11.116001.2]. 

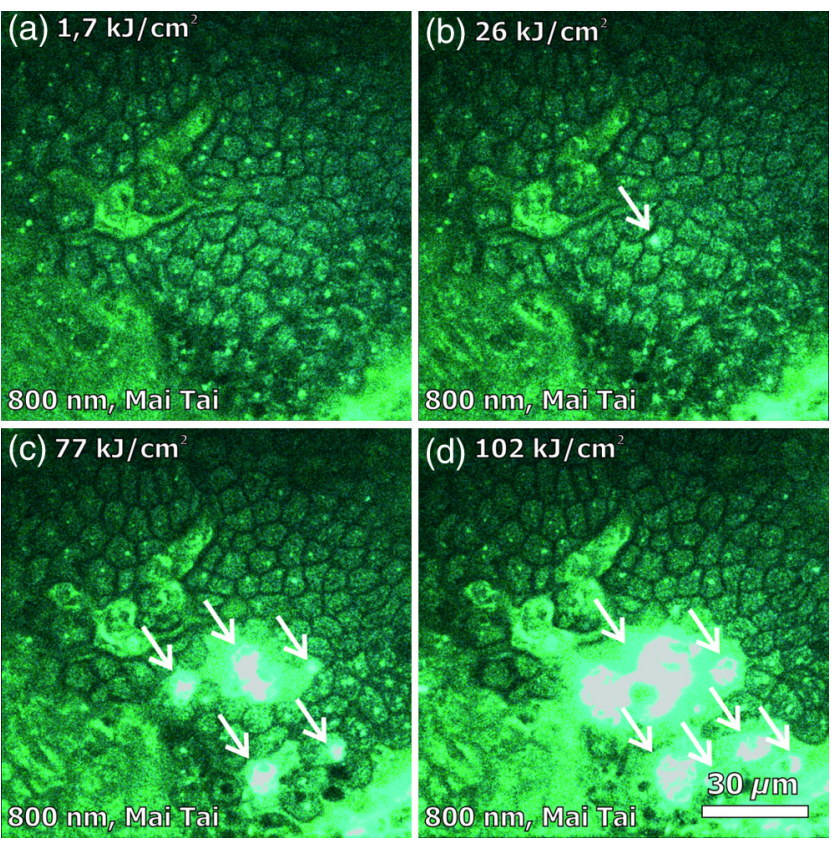

Fig. 5 Four frames from an imaging series, during which a hyperfluorescent lesion in the apical cytoplasm is formed. Tissue was irradiated at $800 \mathrm{~nm}$ with $220 \mathrm{fs}$ pulses. Total radiant exposure for each frame was $1.7 \mathrm{~kJ} / \mathrm{cm}^{2}$. (a) First frame of the imaging sequence without any visible tissue damage. Lysosomes are visible as small bright spots inside the apical cytoplasm of enterocytes, exhibiting a fluorescence red-shift with respect to cytoplasmatic NADH fluorescence. (b) Onset of hyperfluorescence (white arrow) in frame 15 (radiant exposure $26 \mathrm{~kJ} / \mathrm{cm}^{2}$ ) starting from a position where a lysosome was detected before (white arrow). (c) After 45 frames $\left(77 \mathrm{~kJ} / \mathrm{cm}^{2}\right)$, hyperfluorescence (white arrows) has started at multiple sites in the epithelium. (d) Hyperfluorescent lesion after the acquisition of 60 frames $\left(102 \mathrm{~kJ} / \mathrm{cm}^{2}\right)$. Existing lesions had grown in size and more lesions had developed during continued imaging.

(a)

Narrowband pulses $(220 \mathrm{fs})$

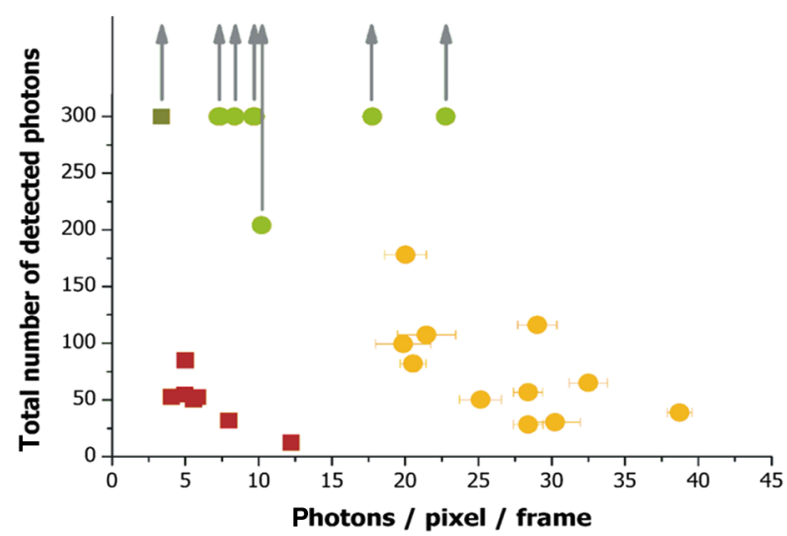

\subsection{Hyperfluorescence and Photodamage}

For different wavelengths and pulse durations, not only the amount of tissue damage but also the damage mechanism may differ. We investigated the tissue damage under different excitation conditions by acquiring image sequences of intestinal villi with the focal plane in the apical or basal cytoplasm of the epithelium. Hyperfluorescence, a strong increase of fluorescence intensity, is one obvious and well documented limit for A2P microscopy. ${ }^{6,19}$

Here, it was generally observed during the acquisition of image sequences of up to 100 frames at a higher excitation flux (Fig. 5). Bright hyperfluorescence appeared first in small areas which could often be assigned to lysosomes [Fig. 5(b)]. Cells that developed hyperfluorescence were immediately destroyed and the affected area spread fast during further irradiation [Figs. 5(c) and 5(d)]. Only at a lower radiant flux could images be taken over a long time without the development of hyperfluorescence.

The total number of fluorescence photons before the onset of hyperfluorescence was determined as a function of the mean excitation flux. Figure 6 shows measurement values for 10 ROI. Each spot represents one irradiation series similar to that shown in Figure 5. The onset of hyperfluorescence was defined by a rise of the emission signal to a level two times above the starting fluorescence intensity. Green spots in the diagram represent an irradiation series with 300 photons detected without hyperfluorescence. These series set a lower limit for the amount of detectable light.

The onset of hyperfluorescence followed a strongly nonlinear relation. In contrast to linear photochemistry, in which a fixed photon budget of incident light is available for imaging, here higher fluorescence rates drastically decrease the total detectable fluorescence. We observed that 220 fs pulses tuned to a wavelength, where $\mathrm{NAD}(\mathrm{P}) \mathrm{H}$ excitation is efficient $(730 \mathrm{~nm})$, could generate more fluorescence photons than (b)

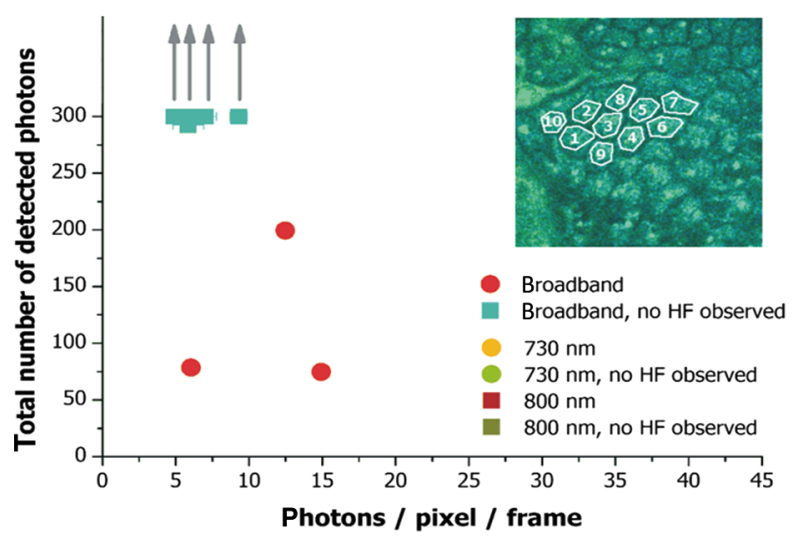

Fig. 6 Total number of detected photons before onset of hyperfluorescence in relation to the fluorescence detection rate for (a) excitation with 220 fs pulses and (b) ultra-broadband pulses. Image sequences of the apical part of the villus epithelium were acquired at different irradiances. The number of detected photons was calculated by multiplying the number of images before the onset of hyperfluorescence with the mean number of photons per pixel. The number of detected photons decreased strongly as fluorescence rate, that is, photons per pixel and frame, increased. Green data points indicate irradiation series, where a total of 300 photons were detected without observation of hyperfluorescence. The inset shows regions of interest in the apical cytoplasm of the villus epithelium that were used to determine the mean fluorescence intensity. The standard deviation shown is the variance between results in the 10 ROls. 
ultra-broadband pulses. With 730-nm excitation, 20 photons per pixel and frame could be measured without observing hyperfluorescence [Fig. 6(a)], whereas with ultra-broadband pulses, the detection rate was limited to 10 photons per pixel [Fig. 6(b)]. When irradiation was conducted with $220 \mathrm{fs}$ at $800 \mathrm{~nm}$, hyperfluorescence was observed after less than 100 photons even with a reduced detection rate of only a few photons per pixel [red squares in Fig. 6(a)].

Hyperfluorescence severely limits A2P microscopy. The strong dependence on the excitation rate actually results in a threshold-like behavior, which limits the image quality in each subsequent frame. With $220 \mathrm{fs} / 730 \mathrm{~nm}$ excitation, a slightly higher signal-to-noise ratio (SNR) $(\sqrt{20}=4.4)$ is achieved than with ultra-broadband pulses (SNR: $\sqrt{10}=3.1$ ). The lower threshold for hyperfluorescence generation could be caused by the shorter pulse duration and/or by a different spectral overlap of excitation and 2-photon absorption spectra.

Comparison with $220 \mathrm{fs}$ excitation at $800 \mathrm{~nm}$ demonstrates the imminent importance of efficient $\mathrm{NAD}(\mathrm{P}) \mathrm{H}$ excitation. Here, the SNR was merely $\sqrt{5}=2.2$. Apparently, longer wavelengths around $800 \mathrm{~nm}$ contribute more strongly to the formation of hyperfluorescence than to autofluorescence. Spectral overlap has a much stronger influence than pulse width. The low damage threshold for ultra-broadband pulses is probably caused by the poor spectral overlap with $\mathrm{NAD}(\mathrm{P}) \mathrm{H}$ absorption rather than by the higher peak irradiance. As a consequence, it is not expected that the onset of photodamage can be mitigated simply by increasing the pulse width. It may be better to decrease the long-wavelength content of the excitation, even at the cost of reduced excitation of red-absorbing flavines and lipopigments. We conclude that increasing the fluorescence-to-damage ratio requires optimization of the excitation spectrum.

\subsection{Recruitment of Immune Cells into the Epithelium as a Sign of Photodamage}

Already at laser powers below the onset of hyperfluorescence, immunological reactions were observed as a first sign of photodamage in the basal part of the epithelium in intestinal villi. Under physiological conditions, the epithelium of small intestinal mucosa contains intraepithelial lymphocytes ${ }^{6}$ but no PMNLs, which include eosinophil and neutrophil granulocytes. Intraepithelial lymphocytes usually reside in the basal part of the epithelium right above the basement membrane and appear dark between the bright enterocytes. This is because lymphocytes have a prominent nucleus which gives no fluorescent signal and only a small rim of cytoplasm. ${ }^{6}$ They move with an approximate speed of $8 \mu \mathrm{m} \mathrm{min}^{-1}$ and have an average diameter of $6 \mu \mathrm{m}$ (Fig. 7, Video 3). Intraepithelial lymphocytes probably play a major protective role against invasion and systemic dissemination of enteric pathogens and commensal bacteria. ${ }^{28}$ In contrast, PMNLs in the epithelium are only expected under pathological conditions. They are attracted after severe tissue damage, such as that caused by laser-induced cavitation, ${ }^{29}$ and can be identified in A2P microcopy by size, speed, and the special shape of their nucleus. ${ }^{29}$ PMNLs, as a sign of sterile inflammation, appeared within the epithelium in the long-time imaging series of the basal part of the epithelium (Figs. 8, 11, Video 4, Video 5, and Video 6) even at radiant flux values below the threshold of hyperfluorescence formation (corresponding to the green spots in Fig. 6). Most of PMNLs show multilobular nuclei and thus probably resemble eosinophil granulocytes, which are abundant in the small intestinal lamina propria

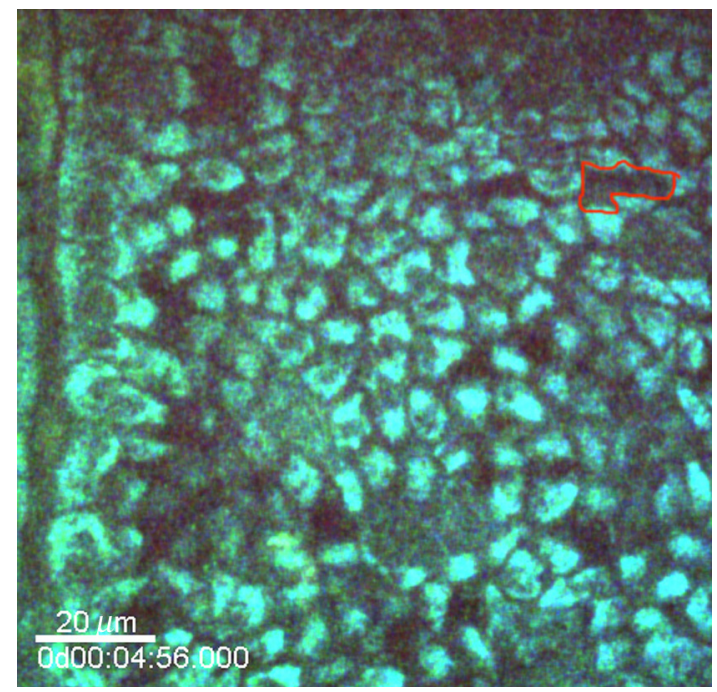

Fig. 7 Time series of the basal cytoplasm of the epithelium with 220 fs laser pulses covering $14 \mathrm{~min}$. Intraepithelial lymphocytes (red outline) move between the enterocytes (Video 3, MPEG, 3.5 MB) [URL: http:// dx.doi.org/10.1117/1.JBO.20.11.116001.3].

beneath the epithelium. They have diameters of $12-15 \mu \mathrm{m}$ and move with average speed of $22 \mu \mathrm{m} / \mathrm{min}$ between the enterocytes (Figs. 8, 11, Video 4, Video 5, and Video 6). We attribute their appearance to light-induced tissue damage and the release of pro-inflammatory cytokines and damage-associated molecular pattern molecules (DAMPs) by the affected cells.

Recruitment of PMNLs was far less pronounced when the basal part of the small intestinal epithelium was imaged with $220 \mathrm{fs} / 730 \mathrm{~nm}$ pulses. In a 100-frame time series of over $14 \mathrm{~min}$, only the movement of intraepithelial lymphocytes above the basement membrane was observed in most of the imaged villi (Fig. 7, Video 3). Only in $17 \%$ of the imaged villi was the invasion of PMNLs visible (Fig. 9). Using ultrabroadband pulses for acquiring images of equal quality, PMNLs appeared in $62 \%$ of the image series (Fig. 9).

In addition to the 14-min time-lapse series, further image stacks of the irradiated villi were taken at single time points after the end of the image series. Not surprisingly, the PMNLs' incidence rate further increased with time. Within $1 \mathrm{~h}$ after the start of imaging, in all of the volumes recorded with ultra-broadband pulses immigrated granulocytes were visible (Fig. 9). In contrast, when using 220 fs pulses, PMNLs were only observed for $40 \%$ of the villi (Fig. 9). Irradiation of the apical cytoplasm of the epithelium seems to less affect the cells.

Here, no PMNLs at all were observed with 200 fs excitation, and they appeared only in $33 \%$ of the villi imaged with ultrabroadband pulses (Fig. 9).

When long imaging series in the basal cytoplasm of the epithelium were recorded with ultra-broadband pulses, PMNLs were recruited much faster into the epithelium. They could be observed already moving between the enterocytes after 6 to $8 \mathrm{~min}$ of continuous imaging (Fig. 11, Video 5). After 16 min of consistent scanning with ultra-broadband laser pulses, considerably more PMNLs were seen in the epithelium than with 200 fs laser pulses (Figs. 8 and 11). Fluorescence increased when these cells probably degranulated and died (Fig. 11 Video 6). The faster appearance and higher incidence of PMNLs for images with ultra-broadband pulses is consistent 

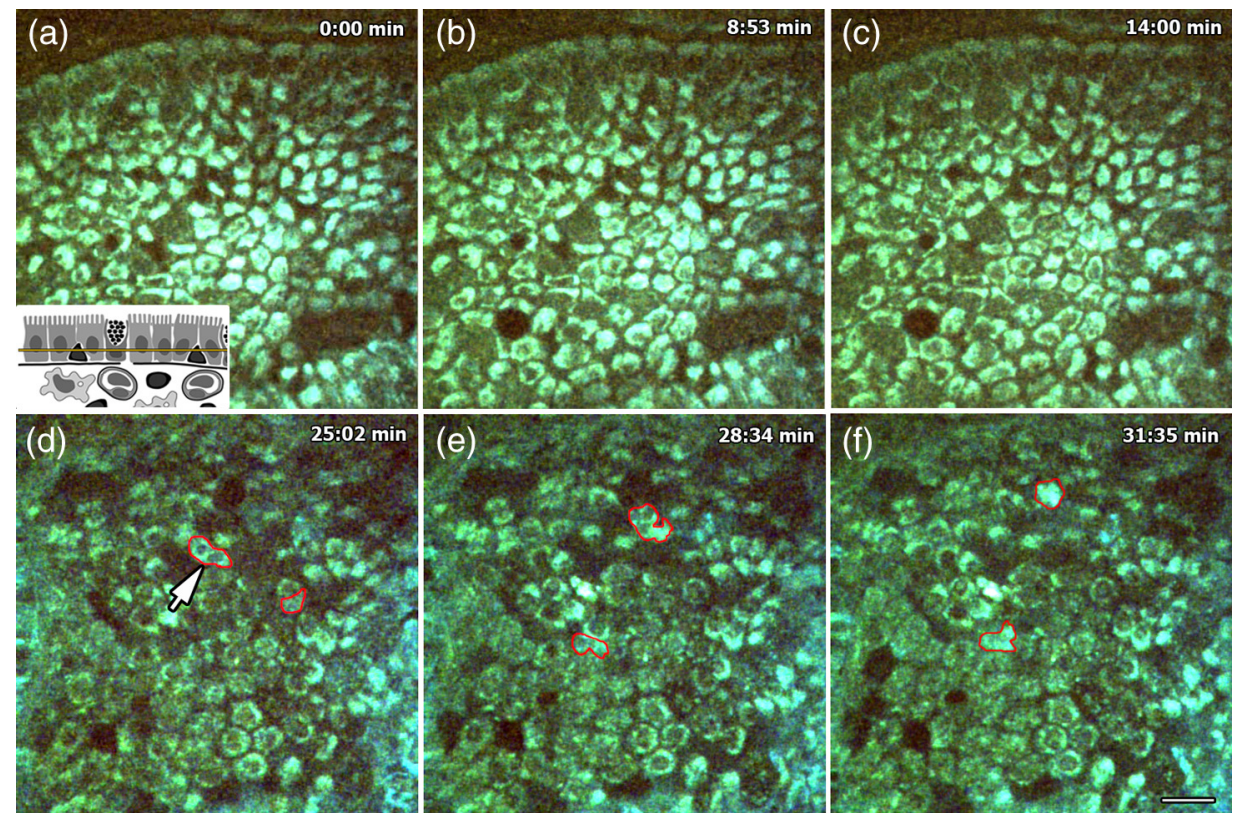

Fig. 8 Time series of the basal cytoplasm of the epithelium with 220 fs laser pulses. Within the first $14 \mathrm{~min},(\mathrm{a}-\mathrm{c})$ no tissue damage can be detected. After $28 \mathrm{~min}$, (d) polymorphonuclear leucocytes (PMNLs) (red outline) can be detected in the epithelium. (d-f) Many of them show multilobular nuclei (arrow) (d-f). Photodamage of the tissue causes also a loss of autofluorescence and blurred cell borders. The yellow line in the inset in (a) shows the imaged plane for the series (scale bar, $15 \mu \mathrm{m}$ ). (d-f) Video 4 shows the time series presented (Video 4, MPEG, 7.46 MB) [URL: http://dx.doi.org/10.1117/1.JBO.20.11 .116001.4].

with a higher degree of other typical signs of tissue damage, such as decreased autofluorecence, blurring of cell borders, and contraction of villi (Figs. 10 and 12).

While PMNLs are not present in the small intestinal epithelium under physiological conditions, they may invade the tissue in response to several chemical mediators. Tissue damage and cellular necrosis lead to the release of "immune triggers" that are typically hidden in quiescent states. In contrast, the regular frequent cell shedding in the villus epithelium is the result of an orchestrated cell death program and is immunologically silent.

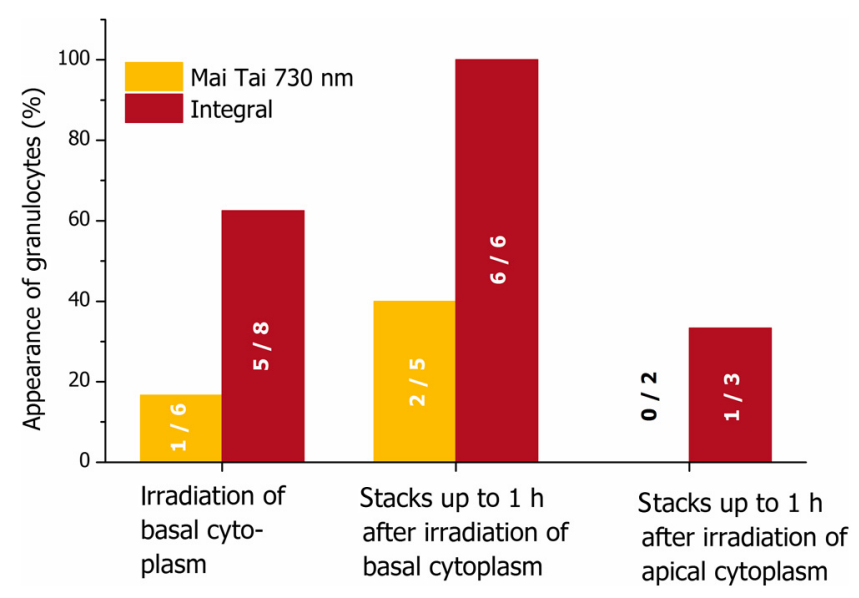

Fig. 9 Ratio between the number of image sets containing granulocytes and total number of datasets. Imaging with ultra-broadband pulses triggered the appearance of polymorphonuclear leucocytes (PMNLs) more frequently compared to imaging with 220 fs pulses at the $730 \mathrm{~nm}$ excitation wavelength.
Various mechanisms produce chemical mediators that can recruit granulocytes to inflammatory sites. Intravital imaging with 2-photon microscopy can lead to the formation of singlet oxygen and reactive oxygen species (ROS) when tissue endogenous fluorophores, such as $\mathrm{NAD}(\mathrm{P}) \mathrm{H}$ and FAD, act as photosensitizers in photo-oxidative processes. ${ }^{30,31}$ ROS are oxygencontaining molecules with unpaired electrons in their outer orbit, that is, metabolites of oxygen that possess strong oxidizing capabilities. In a healthy organism, they are mainly produced by the mitochondrial respiratory chain. At low concentrations, the ROS function as signaling molecules that regulate cell growth, the adhesion of cells toward other cells, differentiation, and apoptosis. ${ }^{32}$ An enhanced production of ROS can pose a severe threat to cells and, if not controlled by sophisticated antioxidant machinery, ultimately leads to cell death. Necrotic cell death is followed by the liberation of a plethora of DAMPs which act as chemical mediators and lead to chemotaxis of eosinophil and neutrophil granulocytes to the site of lesion. ${ }^{33,34}$ For imaging at the same fluorescence brightness, production of ROS seems to be more pronounced with the ultra-broadband pulses. Many more PMNLs were recruited into the epithelium compared to imaging with 220 fs pulses. Granulocytes are among the first blood-borne cells that travel into damaged organs in which they phagocytose particulate material, such as cell debris. During phagocytosis, however, they also generate ROS. This functional response, termed an oxidative burst, contributes to the host's defense, but it can also result in collateral damage of host tissue. Thus, these cells are central to both repair and destruction of tissues following sterile injury. ${ }^{35,36}$ Degranulation of granulocytes, as seen in A2P microscopy with the ultra-broadband laser pulses, resulted in the generation of highly fluorescent material within the tissue, quiescent 

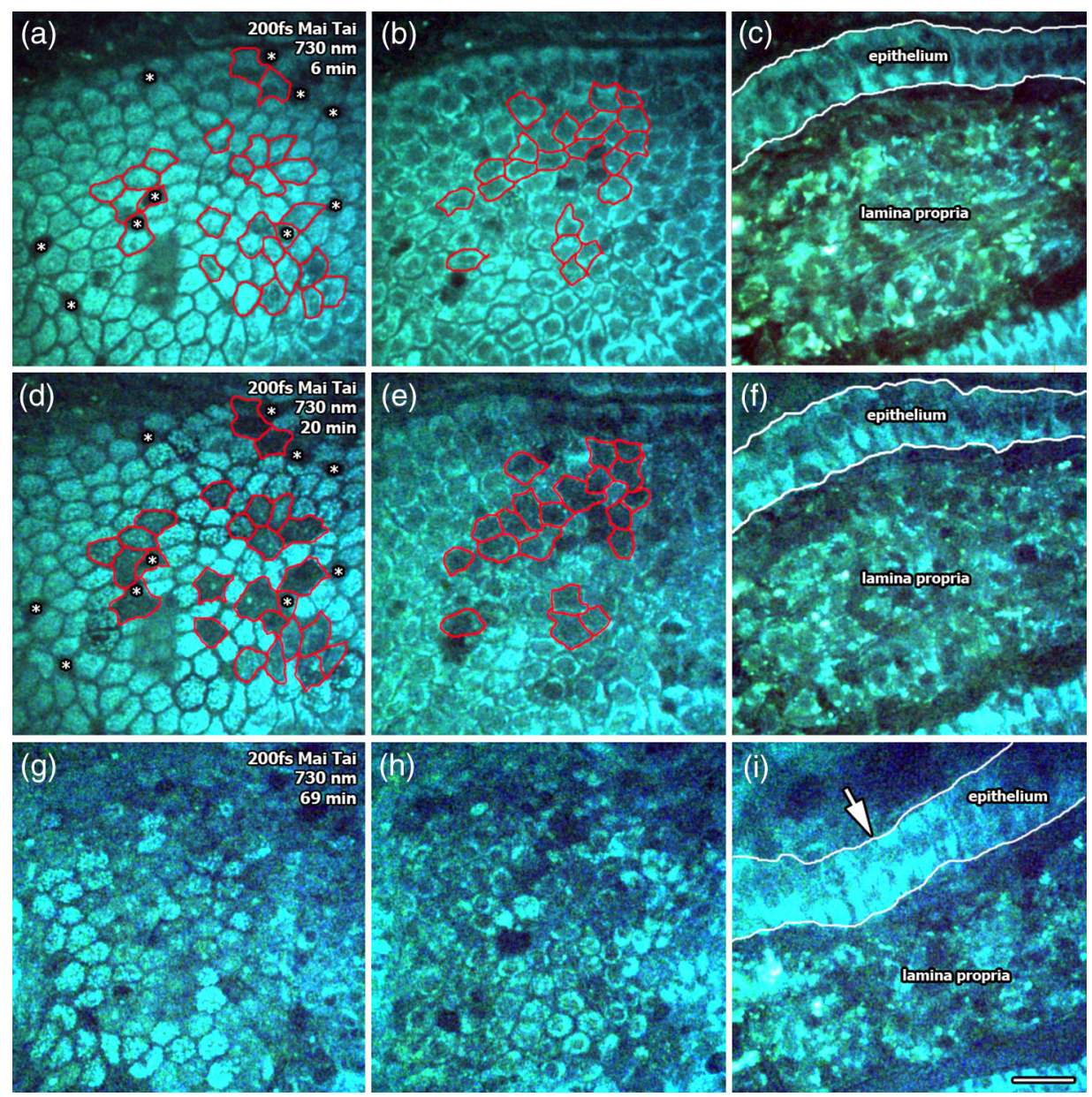

Fig. $10(a, d, g)$ Stack of optical sections through a villus imaged with 220 fs laser pulses at $730 \mathrm{~nm}$ showing the apical cytoplasm, (b, e, h) the section of enterocyte nuclei, and (c, f, i) the lamina propria beneath the epithelium. Within the first $6 \mathrm{~min}(\mathrm{a}-\mathrm{c})$, the tissue remains visually unaffected by the scanning procedure. After $20 \mathrm{~min}(\mathrm{~d}-\mathrm{f})$, the tissue shows the first signs of photodamage. Epithelial cells show loss of autofluorescence as seen by the darkening of the cells (red outline in a, b, d, e). After 69 min ( $g-i)$, the tissue shows massive signs of photodamage, as seen by further darkening of the tissue and blurred cell borders. The villus responds to photodamage with contraction (arrow in i) (scale bar, $15 \mu \mathrm{m}$ ).

PMNLs, and destruction of these immune cells (Figs. 11, 12, Video 6). These highly fluorescent areas are not identical with the described hyperfluorescence, as these areas did not spread during further irradiation and hyperfluorescence was only detected at higher excitation flux.

Invasion of PMNLs into the epithelium has also be observed after UV-A laser nanosurgery of intestinal mucosa, involving bubble formation and localized tissue destruction. ${ }^{29}$ However, just as during long imaging series with 220 fs laser pulses, no degranulation of PMNLs was observed.

Previous experiments in cell culture with longer pulse widths ranging from 120 to 2000 fs showed no dependence of fluorescence yield before cell damage on excitation pulse length. ${ }^{16}$ The results of the present study indicate that this may not hold for ultra-broadband pulses, which excite the autofluorescence in the range from 700 to $900 \mathrm{~nm}$.

\section{Conclusions}

We compared image quality, photodamage, and the occurrence of an inflammatory tissue response in 2-photon microscopy of mouse small intestinal mucosa with excitation at different pulse widths and spectral extents. We chose ultra-broadband pulses for two reasons: first, simultaneous excitation of NADH, FAD, and collagen SHG is achieved in the sample and second, even with compensating only the linear part of the GDD of the optical components with a readily available chirped mirror based precompression stage, a pulse width about an order of magnitude shorter will compensate for the limited spectral overlap. A2P microscopy with 220 fs lasers appears to be a safe novel sectioning technique when imaging duration and excitation flux are kept below certain values. Since tissue damage can be quite subtle and will occur statistically, excitation for imaging should always be set to a minimal level just sufficient to generate the required SNR and an optimal excitation wavelength should be chosen, reducing peak laser intensities and avoiding excitation of potentially phototoxic molecules which do not contribute to the fluorescence signal. Minimal scanning times and high sensitivity detection electronics should be used to ensure maximal detection efficiency. Unnecessarily long time series should be avoided, reducing ROS production and allowing cells to produce sufficient antioxidant substances. Recovery of tissue after photo-oxidative stress is probably limited since larger 

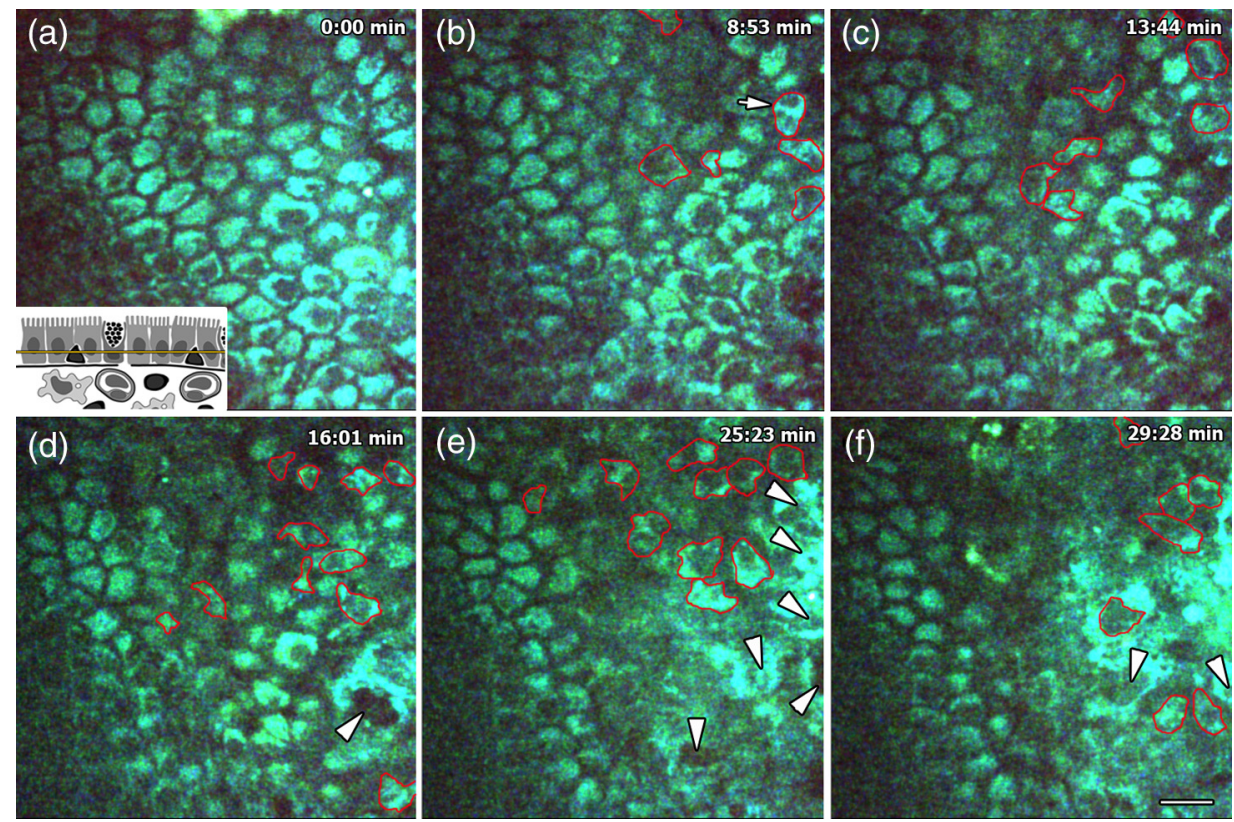

Fig. 11 Time series of the basal cytoplasm of the epithelium with ultra-broadband pulses. After 6 to $8 \mathrm{~min}$ of repeated scanning, the first PMNLs (red outline) appear in the epithelium (b). Many of them show multilobular nuclei (arrow) (b, Video 5; Video 6) Some PMNLs show very strong fluorescence and seem to degranulate (arrowheads). Invasion of leucocytes and degranulation leads to tissue damage, seen by the loss of autofluorescence in epithelial cells. The yellow line in the inset in (a) shows the imaged plane (scale bar, $15 \mu \mathrm{m}$ ). (a-c) Video 5 shows the time series presented. (d-f) Video 6 shows the time series presented (Video 5, MPEG, 9.21 MB; Video 6, MPEG, $10.8 \mathrm{MB}$ ) [URL: http://dx .doi.org/10.1117/1.JBO.20.11.116001.5]. [URL: http://dx.doi.org/10.1117/1.JBO.20.11.116001.6].
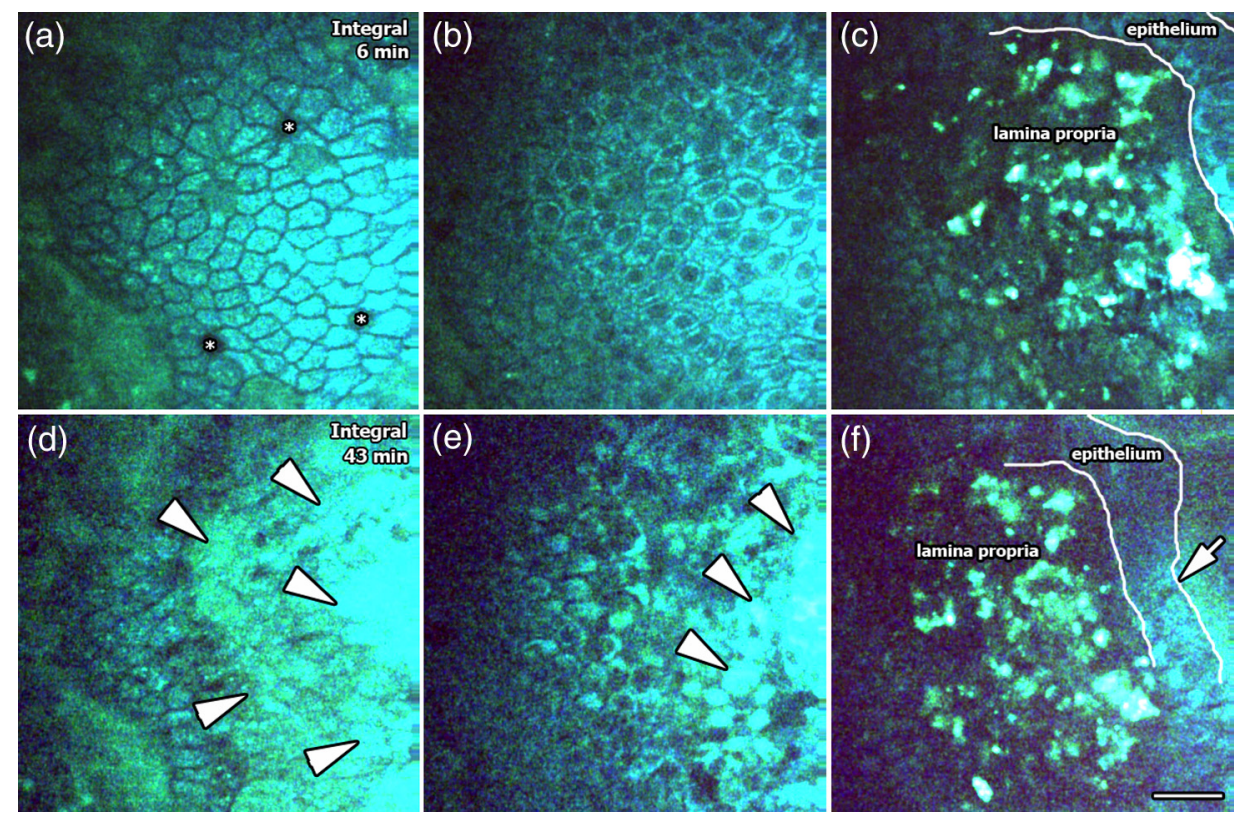

Fig. 12 (a, d) Stack of optical sections through a villus with ultra-broadband excitation showing the apical cytoplasm, $(b, e)$ the section of enterocyte nuclei, and (c, f) the lamina propria beneath the epithelium. $(a-c)$ Within the first 6 min, the tissue remains visually unaffected by the scanning procedure. (d-f) After $45 \mathrm{~min}$, the tissue shows massive signs of photodamage. Highly fluorescent material (arrowheads) is visible above the villus (d) and also in the basal cytoplasm, (e) where the time series (Fig. 11) were done. (d, e) Epithelial cells also show loss of autofluorescence as seen by darkening of the cells. The villus responds to photodamage with contraction (arrow in f) (scale bar, $15 \mu \mathrm{m}$ ). 
disturbances of cell metabolism by strongly absorbing molecules such as $\mathrm{NAD}(\mathrm{P}) \mathrm{H}$ and FAD can exceed the repair capacity of the cell, and toxic effects gradually take place. Switching to a longer wavelength to avoid excitation of endogenous fluorophores might be useful, but needs the application of exogenous fluorophores, thus making it not feasible for clinical diagnosis.

A2P microscopy with ultra-broadband ultrashort femtosecond lasers was not beneficial. Imaging revealed no further information compared to imaging with 200 fs lasers at 730 and $800 \mathrm{~nm}$ and the promise of substantial reduction of excitation flux is only to fulfill with sophisticated dispersion correction. In contrast to a theoretically possible increase of NADH fluorescence, excitation with ultrashort broadband pulses generated less signal for the same excitation power. Although this can be compensated by increased laser output power, the lower threshold of hyperfluorescence effectively reduced the usable image brightness. Furthermore, the immigration of PMNLs into the tissue, a sign of inflammation, is a severe criterion for exclusion from all physiological and immunological questions to be answered with A2P microscopy. Both signs for cell damage were more pronounced at ultra-broadband excitation when the laser power was adjusted for the same level of autofluorescence in the spectral range from 400 to $600 \mathrm{~nm}$.

Besides these acute signs, photodamage limits intravital imaging in animals, and photochemical modifications of the DNA may occur. ${ }^{37,38}$ Three-photon absorption of DNA leads to carcinogenic mutations which are promoted by using shorter pulses. Thymine cyclopyrimidine dimers, a major product of photochemical DNA damage, were generated in cells by a mixture of 2- and 3-photon processes. ${ }^{13}$ They were also detected at low concentration after 2-photon imaging of human skin. ${ }^{39}$ DNA damage is especially of concern for clinical diagnosis or investigating long-term effects in animals, but not for typical intravital imaging setting. Hence, we refrained from investigations in this type of damage. Clinical use of ultra-broadband pulses will, therefore, definitely raise the question of safe pulse widths.

In conclusion, our results stress the importance of choosing optimal excitation to maximize the fluorescence-to-damage ratio. For an optimal parallel excitation at 730 and $800 \mathrm{~nm}$, two lasers should be employed instead of using ultra-broadband pulses. Whether the lack of spectral overlap between excitation and absorption or increased peak power is more responsible for the increased damage cannot be determined from our data. Hence, further experiments with controlled pulse width and spectral phase are needed.

\section{Acknowledgments}

The authors thank Fred Reinholz for the critical review of the manuscript. This work was funded by the German Research Foundation (DFG), (Project Nos. Hu 629/3-2, Kl 2712/1-2, and Vo 470/14-2).

\section{References}

1. W. R. Zipfel, R. M. Williams, and W. W. Webb, "Nonlinear magic: multiphoton microscopy in the biosciences," Nat. Biotechnol. 21(11), 1369-1377 (2003).

2. J. R. McDole et al., "Goblet cells deliver luminal antigen to CD103+ dendritic cells in the small intestine," Nature 483(7389), 345-349 (2012).
3. A. Klinger et al., "Complex morphology and functional dynamics of vital murine intestinal mucosa revealed by autofluorescence 2-photon microscopy," Histochem. Cell Biol. 137(3), 269-278 (2012).

4. E. Dimitrow et al., "Sensitivity and specificity of multiphoton laser tomography for in vivo and ex vivo diagnosis of malignant melanoma," J. Invest. Dermatol. 129(7), 1752-1758 (2009).

5. M. J. Koehler et al., "Non-invasive evaluation of dermal elastosis by in vivo multiphoton tomography with autofluorescence lifetime measurements," Exp. Dermatol. 21(1), 48-51 (2012).

6. R. Orzekowsky-Schroeder et al., "In vivo spectral imaging of different cell types in the small intestine by two-photon excited autofluorescence," J. Biomed. Opt. 16(11), 116025 (2011)

7. W. R. Zipfel et al., "Live tissue intrinsic emission microscopy using multiphoton-excited native fluorescence and second harmonic generation," Proc. Natl. Acad. Sci. U. S. A. 100(12), 7075-7080 (2003).

8. M. Drobizhev et al., "Two-photon absorption properties of fluorescent proteins," Nat. Methods 8(5), 393-399 (2011).

9. C. Wang and A. T. Yeh, "Two-photon excited fluorescence enhancement with broadband versus tunable femtosecond laser pulse excitation," J. Biomed. Opt. 17(2), 025003 (2012).

10. I. Pope et al., "Simultaneous hyperspectral differential-CARS, TPF and SHG microscopy with a single 5 fs Ti:Sa laser," Opt. Express 21(6), 7096-7106 (2013).

11. H. Studier, H. G. Breunig, and K. Konig, "Comparison of broadband and ultrabroadband pulses at $\mathrm{MHz}$ and $\mathrm{GHz}$ pulse-repetition rates for nonlinear femtosecond-laser scanning microscopy," J. Biophotonics 4(1-2), 84-91 (2011).

12. D. Debarre et al., "Mitigating phototoxicity during multiphoton microscopy of live Drosophila embryos in the 1.0-1.2 microm wavelength range," PLoS One 9(8), e104250 (2014).

13. O. Nadiarnykh et al., "Carcinogenic damage to deoxyribonucleic acid is induced by near-infrared laser pulses in multiphoton microscopy via combination of two- and three-photon absorption," J. Biomed. Opt. 17(11), 116024 (2012).

14. A. Vogel et al., "Mechanisms of femtosecond laser nanosurgery of cells and tissues," Appl. Phys. B 81(8), 1015-1047 (2005).

15. A. Hopt and E. Neher, "Highly nonlinear photodamage in two-photon fluorescence microscopy," Biophys. J. 80(4), 2029-2036 (2001).

16. K. König et al., "Pulse-length dependence of cellular response to intense near-infrared laser pulses in multiphoton microscopes," Opt. Lett. 24, 113-115 (1999).

17. H. J. Koester et al., "Ca2+ fluorescence imaging with pico- and femtosecond two-photon excitation: signal and photodamage," Biophys. $J$. 77(4), 2226-2236 (1999).

18. G. Y. Chen and G. Nunez, "Sterile inflammation: sensing and reacting to damage," Nat. Rev. Immunol. 10(12), 826-837 (2010).

19. L. M. Tiede and M. G. Nichols, "Photobleaching of reduced nicotinamide adenine dinucleotide and the development of highly fluorescent lesions in rat basophilic leukemia cells during multiphoton microscopy," Photochem. Photobiol. 82(3), 656-664 (2006).

20. P. Xi et al., "Greater signal and less photobleaching in two-photon microscopy with ultrabroad bandwidth femtosecond pulses," Opt. Commun. 281, 1841-1849 (2008).

21. J. M. D. Cruz, V. V. Lozovoy, and M. Dantus, "Coherent control improves biomedical imaging with ultrashort shaped pulses," $J$. Photochem. Photobiol. A 180, 307-313 (2006).

22. S. Huang, A. A. Heikal, and W. W. Webb, "Two-photon fluorescence spectroscopy and microscopy of $\mathrm{NAD}(\mathrm{P}) \mathrm{H}$ and flavoprotein," Biophys. J. 82(5), 2811-2825 (2002).

23. T. Bölke, "Rauschsignalunterdrückende Rekonstruktion multidimensionaler Bilddaten der intravitalen Zwei-Photonen-Mikroskopie," in Medizinischen Fakultät Friedrich-Schiller-Universität Jena (2013).

24. C. Xu and W. W. Webb, "Measurement of two-photon excitation cross sections of molecular fluorophoreswith data from 690 to $1050 \mathrm{~nm}$," J. Opt. Soc. Am. B 13(3), 481-491 (1996).

25. C. A. Schneider, W. S. Rasband, and K. W. Eliceiri, "NIH Image to ImageJ: 25 years of image analysis," Nat. Methods 9(7), 671-675 (2012)

26. P. Xi et al., "Two-photon imaging using adaptive phase compensated ultrashort laser pulses," J. Biomed. Opt. 14(1), 014002 (2009).

27. V. Pervak et al., "Dispersive mirror technology for ultrafast lasers in the range 220-4500 nm," Adv. Opt. Technol. 3(1), 55-63 (2014). 
28. J. Kunisawa, I. Takahashi, and H. Kiyono, "Intraepithelial lymphocytes: their shared and divergent immunological behaviors in the small and large intestine," Immunol. Rev. 215, 136-153 (2007).

29. R. Orzekowsky-Schroeder et al., "Probing the immune and healing response of murine intestinal mucosa by time-lapse 2-photon microscopy of laser-induced lesions with real-time dosimetry," Biomed. Opt. Express 5(10), 3521-3540 (2014).

30. R. M. Tyrrell and S. M. Keyse, "New trends in photobiology. The interaction of UVA radiation with cultured cells," J. Photochem. Photobiol. B. 4(4), 349-361 (1990).

31. P. E. Hockberger et al., "Activation of flavin-containing oxidases underlies light-induced production of $\mathrm{H}_{2} \mathrm{O}_{2}$ in mammalian cells," Proc. Natl. Acad. Sci. U. S. A. 96(11), 6255-6260 (1999).

32. W. Droge, "Free radicals in the physiological control of cell function," Physiol. Rev. 82(1), 47-95 (2002).

33. R. Lotfi, J. J. Lee, and M. T. Lotze, "Eosinophilic granulocytes and damage-associated molecular pattern molecules (DAMPs): role in the inflammatory response within tumors," J. Immunother. 30(1), 16-28 (2007).

34. Q. Zhang et al., "Circulating mitochondrial DAMPs cause inflammatory responses to injury," Nature 464(7285), 104-107 (2010).

35. G. B. Segel, M. W. Halterman, and M. A. Lichtman, "The paradox of the neutrophil's role in tissue injury," J. Leukoc. Biol. 89(3), 359-372 (2011).

36. S. S. Aceves and S. J. Ackerman, "Relationships between eosinophilic inflammation, tissue remodeling, and fibrosis in eosinophilic esophagitis," Immunol. Allergy Clin. North Am. 29(1), 197-211, xiii-xiv (2009).

37. E. Ferrando-May et al., "Highlighting the DNA damage response with ultrashort laser pulses in the near infrared and kinetic modeling," Front. Genet. 4, 135 (2013).

38. M. K. Daddysman and C. J. Fecko, "DNA multiphoton absorption generates localized damage for studying repair dynamics in live cells," Biophys. J. 101(9), 2294-2303 (2011).

39. F. Fischer et al., "Risk estimation of skin damage due to ultrashort pulsed, focused near-infrared laser irradiation at $800 \mathrm{~nm}$," J. Biomed. Opt. 13(4), 041320 (2008).
Antje Klinger (MD and $\mathrm{PhD}$ ) is working at the Institute of Anatomy, University of Luebeck. Her research Interests are immunological and cellular interactions of cells in the small intestine in physiological and pathological states and their visualization with intravital autofluorescence two-photon microscopy.

Lisa Krapf received her Master's degree in physics from the Universität Bayreuth, Germany. In here PhD thesis at the University of Lübeck, she investigated mechanisms for the uptake of nanoparticle in the small intestine by two-photon microscopy.

Regina Orzekowsky-Schroeder received her PhD 2013 from the University of Lübeck for a thesis on two-photon microscopy and UV laser nanosurgery of murine small intestine. She is now working for a leading company in the field of biomedical technology.

Norbert Koop studied physical technology in Lübeck. After working in industry on applications of laser scanning technology, his group at the University of Lübeck is now dedicated to the application of laser material processing in biomedical technology.

Alfred Vogel is professor of physics and holds the chair of Biomedical Optics at the University of Lübeck. He is fellow of SPIE and of the Optical Society of America. His research group has made pioneering experimental and theoretical contributions to the field of pulsed laser interactions with cells and biological tissues, with special emphasis on plasma-mediated ophthalmic laser surgery, and low-density plasma formation relevant for cell surgery and nonlinear microscopy.

Gereon Hüttmann received a $\mathrm{MSc}$ in physics and a $\mathrm{PhD}$ in physical chemistry. From 1992 to 2005, he was research member and scientific group leader of the Medical Laser Center in Lübeck, Germany. Since 2005 he is leading a research group at the Institute of Biomedical Optics, University of Lübeck. His main research interests are photothermal and photochemical microeffects, optical coherence tomography (OCT) and multiphoton microscopy. 